\title{
MANEJO DE VARIÁVEIS PSICOLÓGICAS NO TRATAMENTO DO CÂNCER EM CRIANÇAS: ALGUMAS CONTRIBUIÇÕES DA PSICONEUROIMUNOLOGIA
}

\author{
PSYCHOLOGICAL VARIABLES MANAGEMENT ON \\ CHILDHOOD CANCER TREATMENT: SOME \\ PSYCHONEUROIMMUNOLOGY CONTRIBUTIONS
}

\author{
Sílvia Maria Gonçalves COUTINHO' \\ Hospitalde Apoio de Brasília \\ Áderson L. COSTA JUNIOR ${ }^{2}$ \\ Universidadede Brasília \\ Suyane KANITZ ${ }^{3}$ \\ Universidadede Brasília
}

\begin{abstract}
RESUMO
Estudos em psico-oncologia pediátrica apontam a necessidade de intervenções multiprofissionais sistemáticas que possam contribuir para a minimização de efeitos adversosproduzidospor diferentes situações do tratamentodo câncera que crianças e familiares são submetidos ou, preparar o paciente para enfrentar contextos identificados como de caráter estressante. $O$ presente artigo apresenta os princípios básicos da psiconeuroimunologia e discute a funcionalidade de algumas contribuições desta área para o desenvolvimento de programas de apoio psicológico à crianças com câncer, que permitam a construção de um repertório comportamental e cognitivo apropriado ao enfrentamento de situações aversivas durante o período de tratamento da doença. Um exemplo de programa de apoio psicológico, desenvolvido em uma unidade de saúde do Distrito Federal é descrito.
\end{abstract}

Palavras-chave: Psicologia da Saúde, psico-oncologia pediátrica, estratégias de enfrentamento, psiconeuroimunologia.

\footnotetext{
(1) Mestre em Psicologia. Funcionária da Fundação Hospitalar do Distrito Federal. Endereço: SaN 202 Bloco 'G' Apartamento 201. 70832-080. Brasília. DF.

(2) Professor Assistente do Instituto de Psicologia da Universidade de Brasília (UnB). Endereço: SaN 211 Bloco 'K' Apartamento 604.70863-110. Brasília. DF. E-mail: aderson@unb.br.

${ }^{(3)}$ Estudante de graduação em Psicologia da Una. Bolsista do PIBIC - UnB/CNPq. Endereço: SHIN ai 2 Conjunto 13 Casa 02. 71510-130. Brasília. DF.
} 


\begin{abstract}
Pediatric psychoncology studies show the need for multiprofessional and systematic interventiqns that may contribute to reduction of adverse effects from different situations on cancer treatment that children and their parents are submitied, to or prepare lhe patient to cape with stress ful contexts. The aim of this paper is to present and dis'cuss Ihe basic psychoneuroimmunology principles and the use of some contribuitions to psychological support programs for children with cancer, allow the acquisition of behavioral and cognitive repertorie suitable for coping with aversive situations during lhe disease treatment período. An example of psychological support program has been developed by a public health unit at Distrito Federal, Brazil.
\end{abstract}

Key-words: Health Psychology, Pediatric Psychoncology, Coping Styles, Ps ychone u roi $\mathrm{mm}$ u nology.

\section{O CAMPO DE CONHECIMENTO DA PSICO-ONCOLOGIA PEDIÁTRICA}

O câncer deve ser tratado como um problema desaúde coletiva, sendo responsável por mais de $20 \%$ das mortes registradas em países em desenvolvimento. Informações atuais revelam que o percentual de morte entre crianças, por motivo de câncer, é inferior a $0,9 \%$ do total de casos mundiais da doença, incluindo adultos e crianças. Outro dado mundial revela $\mathrm{o}$ aumento significativo da incidência média da cura de Leucemia Linfóide Aguda (LLA), principal tipo de câncer incidente em crianças, que passou de $30 \%$ na década de 60, para mais de $70 \%$ na década de 90 (Anderson, 1998; Miller, Young \& Novakovic, 1994).

Com o desenvolvimento de novas modalidades de tratamento, incluindo inovações farmacológicas e tecnologia de informática, observa-se uma tendência ao aumento do número de crianças sobreviventes de câncer. Todavia, apesar de mais eficientes, os tratamentos atuais não são suficientemente eficazes para garantirem eliminação da doença em todos os casos, exigindo a exposição da criança a uma programação detratamerito que' inclui eventos estressantes, tais como: internações hospitalares periódicas, proce- dimentos médicos invasivos, terapêutica farmacológica agressiva e restrições ambientais, o que torna o acompanhamento psicológico do paciente e de sua família, em todas as etapas da doença, um elemento indispensável da assistência prestada.

Definindo-se a oncologia como a área da medicina que estuda o câncer, a psico-oncologia pediátrica pode ser caracterizada como o campo da psicologia da saúde que estuda a influência de fatores psicológicos sobre a etiologia, o desenvolvimento e o tratamento de câncer em crianças. Segundo Gimenes (1996), a psico-oncologia começou a surgir como uma área sistematizada de conhecimento a partir do momento' em que a comunidade científica passou a reconhecer que o aparecimento e a remissão do câncer, poderiam estar intermediados por um conjunto de fatores cuja natureza ultrapassava as condições apenas de natureza biomédica.

Avanços mais significativos em psico-oncologia foram observados a partir da década de 70 , com estudos que investigavam a influência de variáveis de caráter psicossocial e comportamental relacionadas ao controle da dor em situações de procedimentos médicos invasivos, estratégias' de enfrentamento em diferentes circunstâncias de tratamento, medidas de qualidade de vida durante e após 
quimioterapia, eficiência de intervenções multidisciplinares e suporte social de pacientes (Costa Jr., 1999).

Apesar desses avanços, Costa Jr. (1999a), observa que ainda não existe um corpo de conhecimento sistematizado em psico-oncologia e aponta a necessidade do cumprimento de algumas exigências para tal: 1) ampliação do espectro de situações, em oncologia, em que o psicólogo possa estar presente; 2) estreitamento da relação entre a produção de conhecimento na área (com rigor metodológico) e a prestação de serviços especializados em psico-oncologia e; 3) elevação do nível técnico em que se dá a intervenção psicológica, exigindo-se adequação teórico-metodológica dos profissionais ao novo contexto (científico e social) que se instala.

\section{A NOÇÃO DE ENFRENTAMENTO}

No contexto da oncologia, o termo enfrentamento foi utilizado, em meados da década de 40, para designar a diversidade de comportamentos que pacientes com câncer, seus familiares e a equipe médica apresentavam em diferentes circunstâncias da doença.

Uma interessante definição de enfrentamento foi apresentada por Monat \& Lazarus (1985), na qual o enfrentamento era definido como o esforço de adaptação do indivíduo à situações desconhecidas de risco, ameaça ou desafio. Nesses casos, o indivíduo tenta lidar com condições ambientais adversas desenvolvendo novos estilos de comportamento ou adaptando estilos antigos.

Uma breve revisão de paradigmas relacionados ao conceito de enfrentamento, no entanto, aponta a existência de múltiplas orientações teórico-filosóficas, desde a busca de correlações entre fatores de personalidade do paciente e estratégias de enfrentamento, até a especificação de ambientes hospitalares que possam atuar como contingências redutoras de comportamentos de estresse em crianças submetidas compulsoriamente a diversos esquemas de tratamento.

Qualquer que seja a orientação teórica do pesquisador, ressalta-se a necessidade de que as intervenções de caráter psicológico possam promover a minimização de efeitos adversos sobre o comportamento da criança, tais como: isolamento social, depressão, baixa resistência à frustração e irritabilidade. No contexto da psico-oncologia pediátrica, as intervenções devem ser orientadas ao desenvolvimento comportamental da criança, constituindo-se em ajuda adicional para o enfrentamento de experiências potencialmente geradoras de medo e dor, tais como a submissão periódica a procedimentos médicos invasivos.

O ambiente hospitalar deve ser estruturado de modo a oferecer oportunidades pontuais de desenvolvimento à criança, da mesma forma que outros ambientes naturais de cuidados. Programas de acompanhamento psicológico, em psico-oncologia pediátrica, podem incluir atividades de estímulo à socialização, aquisição de habilidades comportamentais, seguimento de limites, regras ejogos de desempenho de papéis, que são experiências tradicionalmente encontradas em instituições que atendem à criança em seu ambiente natural, tais como escolas e creches.

\section{APSICONEUROIMUNOLOGIA}

Ao longo dos tempos, é possível observar várias tentativas de compreensão do papel desempenhado por variáveis intervenientes sobre o processo saúde - doença, incluindo-se a consideração da influência de fatores 
psicológicos sobre o desenvolvimento e tratamento de diferentes doenças.

Pelletier (1997) observa que Hipócrates, no século IV a.C., considerava a saúde como um estado de equilíbrio harmonioso entre mente, corpo e ambiente, sendo a doença, uma resultante de desarmonia entre estes elementos. LeShan (1994), destaca que no Século Ild.C., Galeno descrevia as mulheres, que padeciam de melancolia, como possuidoras de maior predisposição a desenvolver câncer de mama do que aquelas que apresentavam atitudes mais positiva diante da vida. Gimenes (1994), por sua vez, ressalta que na Idade Média(Séc. XI), a Igreja pregava o entendimento da doença como uma decorrência de punição divina ou como um resultado de influências demoníacas que atingiam, mais freqüentemente, pessoas mentalmente enfermas ou espiritualmente vulneráveis. No século XIII d.C., relatos de São Tomás de Aquino também apontavam para a aceitação da existência de relações funcionais entre traços específicos de personalidade e a manifestação de doenças, tais como o câncer e outras doenças crônicas (LeShan, 1994).

A evolução científica e tecnológica do final do Séc. XIX permitiu associar as manifestações patológicas à presença de microorganismos causadores, originando, então o modelo biomédico de saúde, o qual atribuía afenômenos de ordem orgânica(físicos e biológicos) a responsabilidade (ou a causalidade) pelas alterações de processos fisiológicos e a conseqüente ocorrência de enfermidades (O'Leary, 1990).

No Século XX, com os estudos pioneiros de Freud sobre conversão histérica e psiconeuroses, percebeu-se as primeiras inadequações da hipótese de causalidade defendida pelo modelo biomédico. Iniciou-se, então, um movimento no sentido de reintegralizar fatores de natureza psicológica e social à compreensão do processo saúde-doença, dando origem às primeiras proposições de modelos biopsicossociais de atenção à saúde (Guimarães, 1988; Spink, 1992).

Carvalho (1992), observa que apesar de várias décadas de discussão sobre a efetividade de modelos biopsicossociais, as práticas de ensino na área de saúde ainda têm priorizado um modelo biomédico de atendimento; a conseqüência desta prática para a psicologia, é a perpetuação de modelos psicoterápicos de atendimento clínico, enfatizando-se a prestação de serviços em consultórios particulares e $\mathrm{o}$ atendimento focalizado sobre o indivíduo e não sobre um processo social desaúde e doença. Na mesma direção, Spink (1992) destaca que "mudanças recentes na forma de inserção de psicólogos na saúde e,a abertura de novos campos de atuação vêm introduzindo transformações qualitativas na prática que requerem, por sua vez, novas perspectivas teóricas" (p. 11).

Conforme aponta Goleman (1997), a literatura faz referência a estudos que investigam a hipótese da existência de uma possível ligação fisiológica entre a mente e o corpo, incluindo a observação de indivíduos não humanos submetidos a condições ambientais aversivas e as evidências de que os efeitos do estresse se manifestam sobre o sistema imunológico, reduzindo seu grau de eficiência geral.

Preconizando a utilização de modelos biopsicossociais de atenção à saúde, a psiconeuroimunologia levanta a hipótese de que situações ambientais caracterizadas por conteúdo psicológico adverso (tais como o estresse) poderiam produzir alterações no sistema imunológico e neural do indivíduo, de forma a propiciar dois tipos básicos de reações: 1) imunodepressão - alteração em elementos do sistema imunológico e/ou na reatividade destes elementos, tornando o organismo mais suscetível a distúrbios fisiológicos e bioquímicos (Adler \& Cohen, 1993; Bovbjerg, 1991; Cohen \& Rabin, 1998; Kiecolt-Glaser \& Glaser, 1995; 1997; Maier \&Watkins, 1998) e; 
2) imunocompetência - alteração na quantidade ou reatividade de elementos do sistema imune, de forma a maximizar a ação de defesa e impedir distúrbios isiológicos e/ou melhorar a capacidade de combate do organismo contra agressores (Andersen, Kiecolt-Glaser \& Glaser, 1994; Cohen \& Rabin, 1998).

$\mathrm{O}$ experimento pioneiro, que buscava relacionar a influência de fatores psicológicos sobre o sistema imunológico, foi realizado por Ader em 1974 (apud Goleman, 1997). Os dados obtidos com ratos apontavam indícios de que o sistema imunológico daqueles animais havia aprendido uma reação condicionada. $\mathrm{O}$ experimento consistia em um condicionamento clássico, em que os sujeitos eram treinados a responder (em esquema aversivo) à água com gosto de sacarina. A água misturada com sacarina era seguida por uma injeção de ciclofosfamida, que produziria náuseas. Por coincidência, os sujeitos começaram a adoecer e morrer. Observações minuciosas levaram o autor a descobrir que a ciclofosfamida produzia, além das náuseas, o efeito de imunossupressão, caracterizada pela diminuição das células-T do sistema imunológico. Mais adiante, Ader percebeu que, após estabelecido o condicionamento respondente, a ingestão da água com sacarina, dissociada da injeção de ciclofosfamida, também produzia uma imunos supressão.

Segundo Bovbjerj (1991), estudos em psiconeuroimunologia devem procurar descrever os efeitos de fatores psicológicos sobre as atividades do sistema imunológico e, reciprocamente, os efeitos de variáveis imunológicas sobre o Sistema Nervoso Central e, consequentemente, sobre o comportamento, em uma tentativa de especificar mecanismos biológicos que sustentem a hipótese de fatores psicológicos como condicionantes de uma predisposição do organismo ao câncer.

Andersen, Farrar, Golden-Kreutz, MacCallum, Cortney e Glaser (1998), por exemplo, realizaram estudo com objetivo de investigar a influência de fatores psicológicos sobre a etiologia de distúrbios neoplásicos. Os autores avaliaram o sistema imunológico de mulheres portadoras de câncer de mama, submetidas a um contexto potencialmente gerador de estresse -tratamento cirúrgico - incluindo mastectomia total ou parcial. Utilizando uma amostra de mulheres que haviam sofrido cirurgia recentemente, avaliaram a ocorrência de estresse e de pensamentos intrusivos (por exemplo, "eutive sonhos sobre ser um paciente com câncer") ou permissivos (por exemplo, "eu tento não pensar sobre isto") em períodos específicos do tratamento. Efetuaram, ainda, testagem imunológica, procedendo-se à análise bioquímica de células NK (natural killer) e linfócitos T. Os autores observaram que o estresse estava significativamente associado a uma redução nonúmero de células NK e ao aumento de pensamentos intrusivos. Os níveis de estresse eram capazes de predizer uma diminuição significativa na reação das células NK à presença do interferongama (um dos mediadores da resposta imune), concluindo-se que a presença do estresse poderia inibir respostas imunológicas específicas que eram funcionalmente relevantes para o prognóstico do câncer, incluindo a reatividade das células NK e células $T$.

Spiegel, Bloom e Kraemer (1989) já haviam demonstrado que a hipnoterapia, como auxiliar ao tratamento de dor, poderia estar associada ao aumento de taxa de sobrevida de mulheres com câncer de mama. Uma amostra de mulheres com câncer obteve sobrevida de umano e meio a mais, em média, do que a taxa de sobrevida de pacientes do grupo controle. Estudos prospectivos mostraram que a divergência nos índices de sobrevida tinha início, em média, 20 meses depois da intervenção se encerrar, sugerindo que $\mathrm{o}$ efeito da intervenção psicológica era bem mais imediato do que se esperava. 
Em uma revisão bibliográfica sobre a relação entre estresse e doenças infecciosas, Cohen e Williansom (1991) propõem três modelos teóricos de como os estressores poderiam influenciar doenças físicas. O primeiro modelo aponta para o papel do estresse na predisposição da pessoa adquirir uma nova infecção. O segundo modelo, aborda a influência do estresse sobre a duração e a gravidade de uma infecção já existente e o terceiro, descreve como o estresse pode influenciar as sensações físicas e a percepção dos sintomas como doenças e a antecipação da busca de serviços de saúde. Todos os modelos enfatizam a influência do estresse como potencial gerador de doença física ou como elemento de oportunização para o desenvolvimento de transtornos de caráter comportamental.

\section{CONTRIBUIÇÕES DA PSICONEUROIMUNOLOGIA PARA O TRATAMENTO DO CÂNCER}

As evidências de que fatores psicológicos poderiam influenciar o curso de doenças crônicas, favorecerama adoção de modalidades de intervenção psicológica para o enfrentamento de situações aversivas relacionadas ao tratamento do câncer. Carey e Burish (1988) apontaram algumas estratégias utilizadas em situação de quimioterapia, para minimizar o estresse condicionado aos possíveis efeitos colaterais do tratamento. Os autores se referem ao estabelecimento de relações entre a utilização de técnicas específicas (tais como a hipnose, a dessensibilização sistemática, o biofeedback associado ao relaxamento muscular progressivo e as técnicas de distração cognitiva) e a redução de náuseas, vômitos, percepção de dor e elaboração de crenças negativas associadas com a quimioterapia.

A correlação positiva entre fatores adversos de natureza psicológica e a incidência de neoplasias geraram o desenvolvimento de programas de acompanhamento psicológico a pacientes com câncer e seus familiares. Simonton, Mathews-Simonton e Creighton (1987), por exemplo, partindo da premissa de que todo indivíduo influencia ativamente a evolução de seu próprio estado de saúde ou de doença, através de reações psíquicas, crenças cognitivas e respostas comportamentais particulares, defendem que a percepção que o indivíduo elabora acerca de sua doença é fundamental para a obtenção da sua participação ativa e positiva durante o processo de enfrentamento da doença e após encerrado este período. Estes autores propuseram um modelo em que o estresse atuaria como um co-fator no desenvolvimento do câncer. A depressão condicionada por um fator ambiental, ou por um estado de estresse, produziria alterações funcionais sobre 0 sistema límbico do paciente; este último exerceria sua função repressora sobre a atividade hipotalâmica, suprimindo parte da atividade imunológica do organismo, o que por sua vez viria a favorecer 0 surgimento e 0 desenvolvimento de células neoplásicas.

A partir deste modelo, Simonton e Cols. (1987) desenvolveram um programa de intervenção psicológica que, visando a alteração da autopercepção do paciente, geraria um clima geral de esperança positiva no paciente; esta percepção seria registrada pelo sistema límbico que atuaria no sentido de favorecer o incremento da atividade imunológica e, conseqüentemente, maximizar a resposta orgânica para o combate ao câncer. Este programa é composto por um conjunto de atividades que incluem: relaxamento e visualização, manejo de benefícios secundários da doença, estabelecimento de objetivos e metas de vida, planejamento de atividades físicas e intervenção psicossocial junto à família.

O primeiro passo do programa é a identificação de fatores estressores que o 
paciente enfrenta (e que podem estar influenciando funcionalmente o processo saúde-doença). Os autores observam que não se deve gerar culpa no paciente, mas tentar mobilizar uma mudança atitudinale liberar energia para lutar simbolicamente contra a doença. Depois, é necessário que se mostre ao paciente que benefícios secundários, proporcionados pela situação de doença, podem ser obtidos.

O relaxamento e a visualização constituem o instrumental técnico do programa e têm por objetivo preparar o organismo para o início do processo de reação ao câncer e aumentar a eficiência potencial do tratamento médico (em andamento). O paciente vai aprender a elaborar imagens mentais do medicamento destruindo as células cancerosas e de células brancas combatendo estas últimas, por exemplo. Estes procedimentos proporcionam oportunidades para que o paciente seja treinado em respostas de autocontrole, que auxiliama liberação de estresse e tensão, entendidos como fatores facilitadores do desenvolvimento de câncer.

Um dos principais objetivos do programa é o estabelecimento de metas de vida em períodos específicos de tempo, após o início do tratamento da doença. O programa deve permitir que os pacientes possam se apegar a elementos essenciais de suas próprias vidas, reforçando suas expectativas de sucesso de tratamento a partirda reestruturação cognitiva de conceitos como recaída e morte. Fazem parte do programa, também, um conjunto de exercícios físicos, para liberar a tensão e ampliar o repertório de comportamentos dos pacientes para enfrentarem situações de dificuldades individuais, tais como lidar com experiências de dor e expectativas de medo.

Por fim, o programa prevê uma atenção especial às necessidades da família do paciente. Matthews-Simonton (1990) propõe um conjunto de estratégias específicas para lidar com paciente e com a família, incluindo:
1) saber respeitar o limite de cada indivíduo, uma vez que o estresse dos familiares é prejudicial ao paciente; 2) constituir um grupo familiar integrado, em que todos os membros possam expressar afetividades e cognições; 3) não superproteger o doente, uma vez que esta prática impede (ou pelo menos dificulta) a mobilização de recursos do próprio paciente; 4) manter as atividades de vida diária o mais próximo do que era antes da doença, visto que os familiares devem estar prontos a ajudar quando for preciso e isto só é possível se eles não estiverem desgastados pelas mudanças já ocorridas; 5) o paciente deve participar de todas as decisões da equipe de saúde, que devem ser integralmente respeitadas pelos membros familiares; 6 ) os familiares devem realizar exercícios de visualização, sempre que possível, em conjunto com o paciente, o que facilita a expressão de sentimentos relacionados às experiências vivenciadas.

Outro programa de apoio psicológico a pacientes com câncer, elaborado por LeShan (1994), propõe o manejo de variáveis relacionadas as estruturas de personalidade $e$ ao repertório de comportamentos emocionais de indivíduos com câncer. O autor destaca três aspectos fundamentais da personalidade de pacientes com câncer: 1) presença de um marcante auto-desprezo - indivíduos que não valorizam suas realizações, não gostam de si mesmos e não acreditam no próprio sucesso; 2) incapacidade de demonstrar repertório de hostilidade em auto-defesa, embora apresentem respostas freqüentes de agressão em defesa dos direitos de outros; 3 ) sentimentos de desespero que resultam dos dois primeiros fatores, e que se tornam elementos básicos de vida. Este desespero não decorre do desenvolvimento do câncer, mas é entendido como um fator de constituição emocional.

Observa-se que o programa desenvolvido por LeShan(1994) pretendedespertar recursos (intrínsecos) da própria pessoa e liberar forças que possam fazer com que a pessoa conheça 
a si mesma. O programa é baseado no pressuposto de que é possível reagir a acontecimentos negativos de forma mais positiva e inclui princípios de relaxamento e de visualização.

No Brasil, Falcone (1995) propõe um programa de atendimento psicológico a pacientes com câncer que se fundamenta em princípios filosóficos da terapia cognitivo-comportamental (TCC). O programa inclui quatro fases distintas: a primeira visa a avaliação de dados físicos e emocionais do paciente, incluindo a utilização de instrumentos específicos para a demanda comportamental de cada paciente, tais como: medidas de depressão, estratégias de enfrentamento para avaliação de situações promotoras de estresse e auto-registro de situações desagradáveis vivenciadas pelo paciente.

$\mathrm{Na}$ segunda fase, é proposta uma abordagem psicossocial na qual se apresenta ao paciente os fundamentos da TCC, ressalta-se o papel ativo do paciente durante o processo detratamento e aponta-sea influência de seu estado psicológico sobre a evolução da doença. Na terceira fase, são introduzidas técnicas de reestruturação cognitiva, buscando-se a modificação de pensamentos automáticos que geram ansiedadee depressão, e o treinamento em solução de problemas, possibilitando o desenvolvimento de estratégias de enfrentamento mais eficientes. $\mathrm{Na}$ quarta fase propõe-se a avaliar os efeitos do tratamento e utilizar os dados obtidos em fases anteriores. Esta fase inclui, também, uma entrevista final que busca identificar mudanças comportamentais e cognitivas percebidas pelo próprio paciente ao longo do programa.

Também no Brasil,o Programa Simonton, para adultos, foi introduzido em São Paulo e vem sendo aplicado, com adaptações, pelo CORA - Centro Oncológico de Recuperação e Apoio - desde 1986, com resultados significativos.

\section{O MANEJO DE VARIÁVEIS PSICOLÓGICAS DURANTEO TRATAMENTO DO CÂNCER EM CRIANÇAS: UM EXEMPLO}

Com crianças em tratamento de câncer, um programa fundamentado nas orientações teórico-filosóficas de Simonton e Cols. (1987) e de LeShan (1994), além de princípios gerais de psiconeuroimunologia e de psicologia cognitiva,vem obtendo resultados promissores junto a uma unidade da rede de saúde do Distrito Federal.

Tendo por objetivos gerais propiciar um atendimento personalizado, humano e de qualidade às crianças com câncer e suas famílias, além de estimulá-los a desempenhar um papel ativo no processo de tratamento, bem como promover o auto-conhecimento das necessidades psicossociais da criança, os programa inclui sessões planejadas (com periodicidade semanal ou quinzenal) que utilizam os recursos técnicos do relaxamento, da visualização ativa e do desempenho psicodramático de papéis. As manipulações ambientais programadas durante as sessões têm por objetivo permitir a aquisição e a manutenção de comportamentos favoráveis ao sucesso do tratamento médico, demonstrando-se as relações funcionais que se estabelecem entre a intervenção profissional em grupo e os efeitos sobre o repertório de comportamentos exibidos pela criança após encerrado o programa.

As atividades programadas pretendem fazer com que a criança construa e possa dispor de um repertório de comportamentos apropriados a enfrentar diversas situações de caráter aversivo durante o período de tratamento da doença. Levanta-se a hipótese de que a manipulação de conteúdo cognitivo, durante as sessões do programa, permitem que a criança possa reestruturar suas percepções acerca de diferentes elementos relacionados à doença e ao tratamento, incluindo-se, por exemplo: 1) descoberta de 
benefícios secundários da doença; 2) estimulação da expressão de sentimentos negativos relacionados à doença; 3 ) redefinição dos conceitos de morte, dor, doença e autoconceito. A reestruturação destas percepções passa a controlar o comportamento da criança em diferentes situações relacionadas ao tratamento da doença.

Sugere-se que a participação no programa, incluindoas oportunidades à criança para conhecer e manejar seus recursos pessoais de enfrentamento à doença e para perceber, compreender e controlar sentimentos e emoções relacionados à doença, atue como situações estabelecedoras de ação prolongada, úteis a diferentes situações de tratamento que ainda serão dispostas à criança no futuro.

As estratégias de intervenção propostas pelo programa visam a possibilidade de obter maior controle funcional sobre as contingências do ambiente, implicando a promoção do desenvolvimento de ambientes hospitalares planejados às necessidades cognitivas e comportamentais de seus usuários, de modo a oferecer às crianças e adolescentes que passam pelo tratamento de câncer, oportunidades de participação em atividades orientadas à estimulação de habilidades próprias a cada estágio do desenvolvimento, bem como, a canalização e mobilização de habilidades comportamentais de auto-ajuda que favoreçam o processo de enfrentamento da doença (em diferentes momentos do tratamento) e atuem como terapia complementar ao tratamento médico.

\section{REFERÊNCIAS BIBLIOGRÁFICAS}

ADLER, R. \& COHEN, N. (1993). Psychoneuroimmunology: Conditioning and Stress. Annual Review de Psychology, 44, 53-82.

ANDERSEN, B.L, KIECOLT-GLASER, J.K. \& GLASER, R. (1994). A biobehavioral model of cancer stress and disease course. American Psychologist, 49, 389-404.

ANDERSEN, B.L, FARRAR, W.B., GOLDENKREUTZ; KURT, LA., MACCALLUM, R., CORTNEY, M.E. e GLASER, R. (1998). Stress and immune responses after surgical treatment for regional Breast Cancer. Journal of Ihe Nacional Cancer Institute, 90(1), 30-36.

ANDERSON, B. (1998). Stress reduction: Three trials test its impact on breast cancer progression. Journal of The National Cancer Institute, 90(1),12-14.

BOVBJERG,D.H.(1991)Psychoneuroimmunology: Implications for Oncology? Cancer, 67(3), 828-832.

CAREY, M.P. \& BURISH, T.G. (1988). Etiology and treatment of the psychological side effects associated with cancer chemotherapy: A critical review and discussion. Psychological Bulletin, 104, 307-325.

CARVALHO, R.S. (1992). A formação em psicologia para o trabalho na saúde pública. Em: F.C.B. Campos (Org.). Psicologia e Saúde: repensando práticas. (pp. 2540). S. Paulo: Hucitec.

COHEN, S. \& WILLIANSOM, G.M. (1991). Stress and infectious disease in humans. Psychological Bulletin, 109, 5-24.

COHEN, S.\& RABIN, B.S. (1998). Psychologic Stress, Immunity, and Cancer. Journal of Ihe National Cancer Institute, 90(1), 3-4.

COSTA JR., A.L (1999). Psico-oncologia e manejo de procedimentos invasivos em Oncologia Pediátrica: uma revisão de literatura. Psicologia: Reflexão e Crítica, 12(1), 107-118.

COSTA JR., A..L. (1999a). Psico-Oncologia pediátrica: subsídios para intervenção profissional. Pediatria Moderna, 36(6), 442-446. 
FALCONE, E. (1995). Projeto de atendimento psicológico a pacientes com câncer. Psicologia Argumento, 17(1), 9-32

GIMENES, M.G. (1994). Definição, foco de estudo e intervenção. Em: M.M.J. Carvalho (Org.), Introdução à Psiconcologia. (pp. 35-56). Campinas, SP: Editorial psy II.

GIMENES, M.G. (1996). Retrospectiva e perspectiva da Psiconcologia no Brasil. Anais do III Encontro e I Congresso Brasileiro de Psico-Oncologia. Sociedade Brasileira de Psico-Oncologia: São Paulo, SP (pp. 01-02).

GOLEMAN, D. (1997).Oque é medicina mente -corpo? Em: D. Goleman \&J. Gurin (Orgs.), Equilíbrio mente - corpo. (pp. 03-14). Rio de Janeiro: Editora Campus.

GUIMARÃES, S.S. (1988). A Hospitalização na Infância. Psicologia: Teoria e Pesquisa, 4(2), 102-112

KIECOL T-GLASER, J.K \& GLASER, R(1995). Psychoneuroimmunology and health consequences: Data and shared mechanisms. Psychosomatic Medicine, 57, 269-274.

KIECOLT-GLASER, J.K \& GLASER, R (1997). Mente e imunidade. Em: D. Goleman \& J. Gurin (Orgs.), Equilíbrio mente - corpo. (pp. 33-52). Riode Janeiro: Editora Campus.

LESHAN, L (1994). Brigando com a vida. São Paulo: Summus Editorial.

MAIER, S. F. \& WATKINS, L.R (1998). Cytokines for Psychologists: Implications of Bidirecional Immune-to-Brain Communicationfor Understanding Behavior, Mood, and Cognition. Psychological Review, 105(1):83-107.

MATTHEWS-SIMONTON, S. (1990), A Família e a cura. São Paulo: Summus Editorial.

MILLER, RW., YOUNG, J.L. \& NOVAKOVIC, B. (1994). Childhood cancer. Cancer, 75 , 395-405.

MONAT, A \& LAZARUS, RS. (1985). Stress and coping - an anthology (pp. 12-36). New York: Columbia University Press.

O' LEARY, A. (1990). Stress, emotion, and human immune function. Psychological Bulletin, 108,363-382.

PELLETIER, K.R (1997). Entre a mente e o corpo: estresse, emoções e saúde. Em: D. Goleman \& J. Gurin" (Orgs.)", Equilíbrio mente-corpo. (pp. 15-31). Rio de Janeiro: Editora Campus.

SIMONTON, O.C., Matthews-Simonton, S. \& Creighton, J.L (1987). Coma vida de novo. São Paulo: 'Summus Editorial.

SPIEGEL, D., BLOOM, J.R \& KRAEMER, H.C. (1989). Eftect of psychosocial treatment on survival of patients with metastatic breast cancer. Lancet, 1, 888-901.

SPINK, M. J. P (1992). Psicologia da Saúde: A estruturação de um novo campo de saber em: F.C.B Campos (Org.), Psicologia e saúde: Repensando Práticas. (pp. 11-23). São Paulo: Hucitec. 